\title{
Engine calibration: multi-objective constrained optimization of engine maps
}

\author{
Hoël Langouët · Ludovic Métivier • \\ Delphine Sinoquet • Quang-Huy Tran
}

Received: 31 October 2008 / Accepted: 31 January 2011 / Published online: 5 April 2011

(C) Springer Science+Business Media, LLC 2011

\begin{abstract}
We present two new approaches to address the optimization problem associated with engine calibration. In this area, the tuning parameters are traditionally determined in a local way, i.e., at each engine operating point, via a single-objective minimization problem. To overcome these restrictions, the first method we propose is able to cope with several objective functions simultaneously in the local formulation. The second method we put forward relies on a global formulation, which allows the whole driving cycle to be taken into account while remaining single-objective. At the practical level, the two methods are implemented by combining various existing techniques such as the LoLiMoT (Local Linear Model Tree) parameterization and the MO-CMA-ES (Multi-Objective Covariance Matrix Adaptation Evolution Strategy) algorithm. A better compromise appears to be achieved on real case applications.
\end{abstract}

Keywords Engine calibration - Response surface $\cdot$ LoLiMoT $\cdot$ Multi-objective optimization · Evolutionary algorithm

\section{Nomenclature}

Abbreviations (by alphabetical order)

CMA-ES Covariance Matrix Adaptation Evolution Strategy

LoLiMoT Local Linear Model Tree

MO-CMA-ES Multi-objective CMA-ES

NEDC New European Driving Cycle

OP Operating Points

RBF Radial Basis Function

SQP Sequential Quadratic Programming

H. Langouët · L. Métivier · D. Sinoquet $(\bowtie) \cdot$ Q.-H. Tran

Département Mathématiques Appliquées, IFPEN, 1 et 4 avenue de Bois Préau,

92852 Rueil-Malmaison Cedex, France

e-mail: delphine.sinoquet@ifpen.fr 


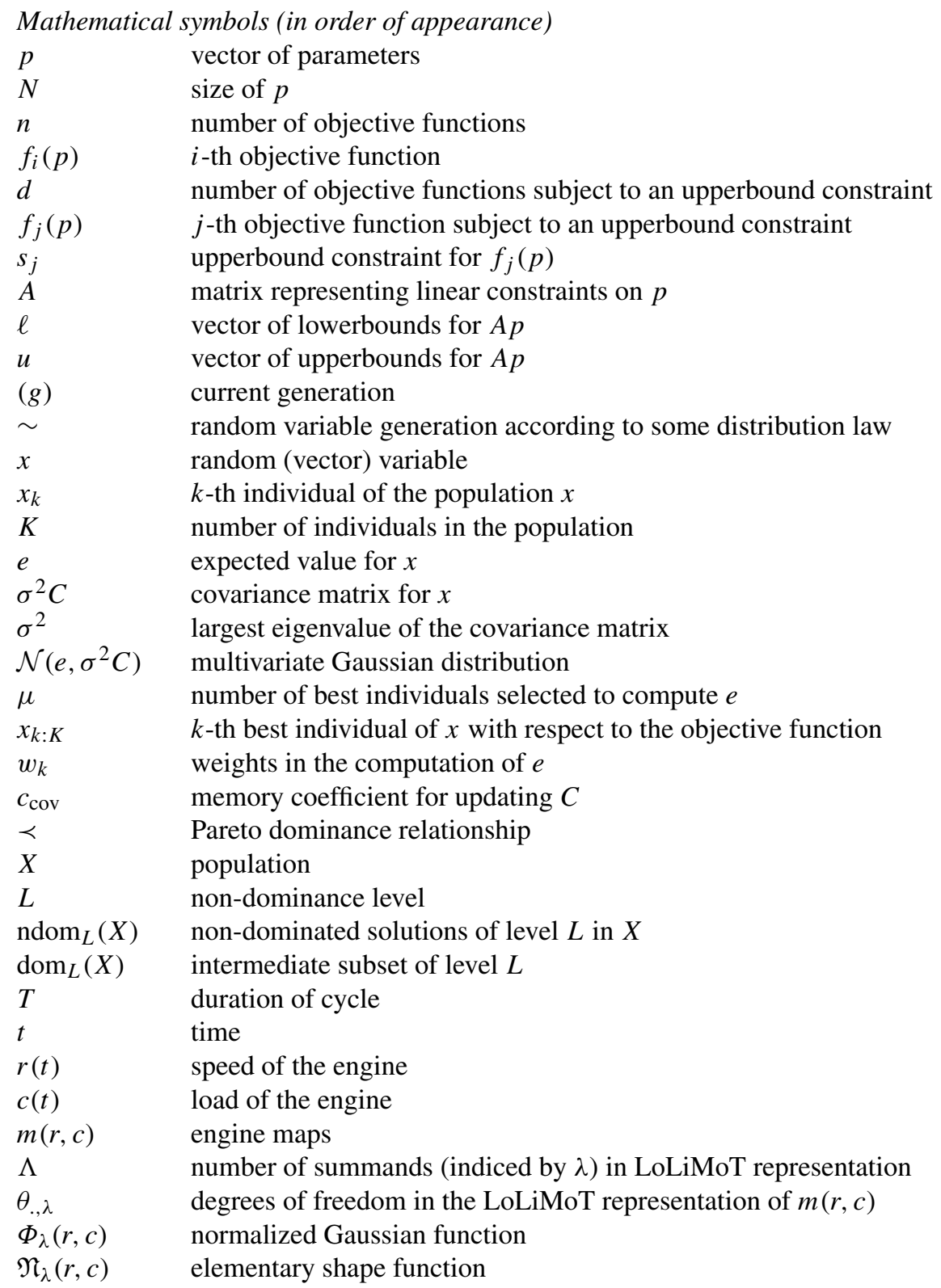

\section{Introduction}

Engine calibration consists in fulfilling the engine tuning maps that are used in engine controls of the vehicle, i.e., in defining the optimal tuning of parameters used by engine control strategies. Due to the highly increased number of these parameters (especially for diesel engines but spark ignition engines are following the same trend) 
and the reduction of the development schedule available for the calibration process, manual tuning of engine parameters is now replaced by mathematically assisted calibration process. Such a process is based on the design of experiments with associated modeling methods, which allows us to reduce the number of tests used to build engine response models depending on engine control parameters, and optimization techniques to determine the optimal settings within the model definition domain (Röpke et al. 2005; Hafner and Isermann 2001; Seabrook et al. 2007; Castagné et al. 2008; Sampson and Sheridan 2007). In order to perform the tests in a more productive way, these mathematical techniques are generally associated with test automation, requiring well controlled measurement methods and reliable test equipments.

The classical approach is based on a local statistical modeling of the engine responses at a limited set of operating points (given engine speed and engine torque or load) from experimental measurements at engine test bench. Then, for each operating point, a local optimization of these models is performed. This optimization problem is often a bi-objective problem which is transformed into a mono-objective problem with a weighted sum of the objectives. The choice of the weights being far from obvious, several trials are often necessary to find an acceptable solution (Das and Dennis 1998; Leyffer 2009). In this paper, we propose to adapt the MultiObjective Covariance-Adaptation Evolution Strategy method (MO-CMA-ES) proposed by Hansen and Ostermeier (1996) to the problems arising in this application: linear and nonlinear constraints are introduced via a suitable penalty procedure.

As pointed out by many previous works (Castagné et al. 2008; Buzy et al. 2005; Schmied 2003), the local approach leads to several difficulties: the definition of the objectives of the local optimization at each operating point is cumbersome, the synthesis of the resulting local optima in engine maps (maps that are implemented in the engine control unit) leads to a difficult smoothing phase that usually ends up with a deterioration of the optimized operating points. Moreover, this method is time-consuming due to the modeling and the optimization phases for each operating point (Castagné et al. 2008). In this respect, Schmied (2003) proposed a global optimization approach which directly optimizes the engine maps on the whole driving cycle instead of the individual optimization of each engine operating point. In one single step, his method optimizes all the chosen operating points and directly delivers the engine maps that are implemented in the unit control of the vehicle. Nevertheless, the implementation of this approach is difficult due to the large number of unknowns of the optimization problem and the number of smoothing constraints to ensure feasibility and drivability (Neßler et al. 2006; Schloßer et al. 2007). To circumvent these difficulties, we advocate the use of an adapted parameterization of the maps based on LoLiMoT models (Nelles 2001; Hafner and Isermann 2001), which allows a priori information on the engine maps to be incorporated naturally and the number of parameters to be reduced significantly.

Section 2 recalls the classical steps of the calibration process, then discusses the difficulties that have motivated our study. In Sect. 3, we outline the Multi-Objective Covariance-Adaptation Evolution Strategy method. Finally, in Sect. 4, the map optimization of the whole driving cycle is described. The potential of the two methods is illustrated by an application on a real calibration case for a turbo-charged commonrail diesel engine. 


\section{Engine calibration}

\subsection{Sketch of the engine calibration process}

The emission calibration workflow is classically divided into three steps (Röpke et al. 2005):

(1) a preliminary phase consists in choosing a sample of operating points (referred to as OP in the following) to be studied and emissions targets associated with each $\mathrm{OP}$, these targets being called allocations hereafter.

(2) the optimization of engine responses on each OP according to these targets,

(3) the fulfilling of the maps with a smoothing step between these optimal settings.

The preliminary step deduces from a simple model of the vehicle the trajectory of the driving cycle in the engine speed-load operating domain (see Fig. 1): the transient effects on the accelerations of the cycle are neglected and the cycle is thus considered as a sum of stabilized points. A limited number of specific Operating Points are chosen to represent the cycle in the engine working range. Figure 1 gives an example of NEDC (New European Driving Cycle) simulation and a selection of 17 OPs. The cumulated levels of pollutant emissions along the cycle are computed as weighted sums of the pollutant emissions for each chosen OP.

Phase 2 consists of five steps:

(i) defining the domain of variations of the engine control parameters: this is an essential step of the process as it defines the validity domain of the models. The complexity of the models to be used for engine response depends on the size of this domain: for tiny domains low order polynomials (second order) are usually sufficient to accurately model engine responses. However, choosing too small domains leads to difficulty in coherently fulfilling sub-optimal engine maps (Castagné et al. 2008).

(ii) building the test matrix: various types of experimental designs can be used to build a test matrix: D-Optimal, space filling... The choice of the type of design as well as the number of tests to be done are directly correlated with the assumed complexity of the model and thus with the size of the considered domain. D-Optimal test designs are often used with hyper-cubic tiny domains (Röpke et al. 2005).

(iii) running this test matrix on the test bench: as the tests are predefined, the experiments can be performed in an automated way, which drastically improves the productivity of the global process. In this case, special attention must be paid to the validation of the experimental data.

(iv) modeling the engine responses: the type of mathematical models depends on the complexity of the engine responses and on the size of the domain. In practice, the engineer often prefers to limit the parameter variations in order to avoid critical engine tunings that may lead to difficulty to realize the experiment at the test bench. However, as already mentioned, reducing the size of the parameter variations may lead to difficulties to build coherent optimal engine maps from the obtained optimal settings in those tiny domains. Often, low order polynomial functions are used coupled with D-optimal design of experiments. For some 
Fig. 1 Simulation of the cycle from European legislation (NEDC: New European Driving Cycle) and selection of operating points
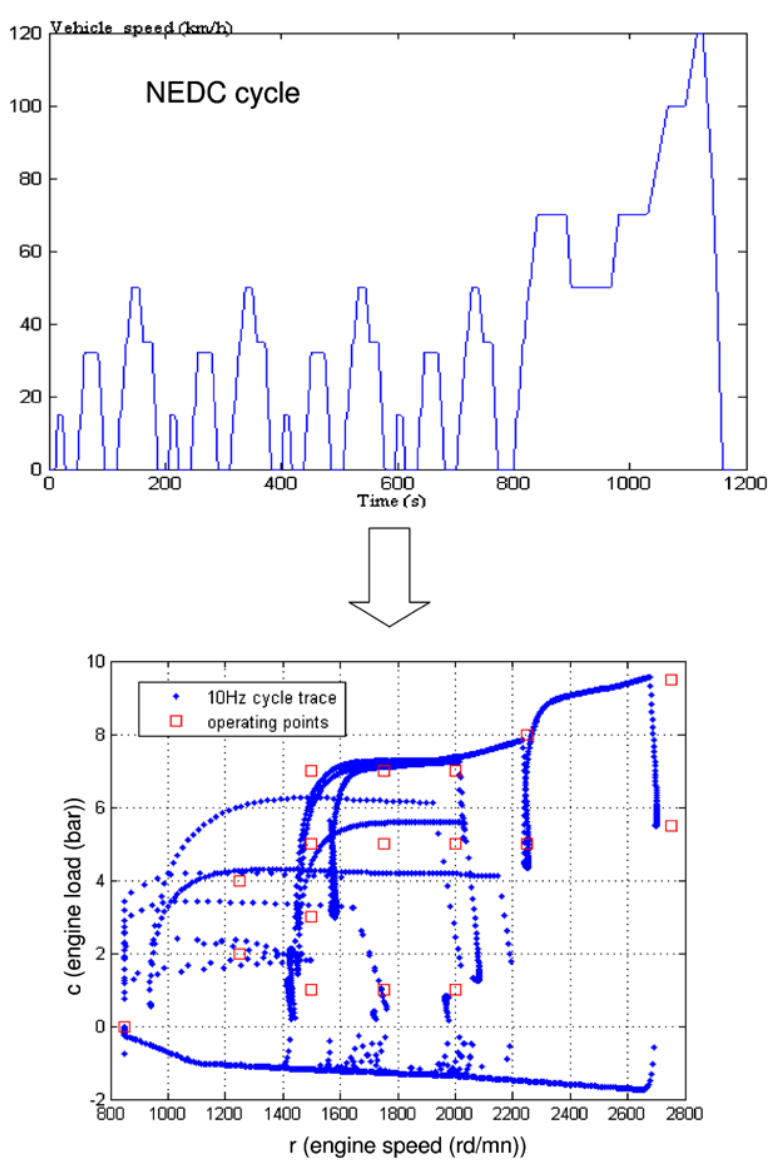

response as $\mathrm{HC}$ or $\mathrm{CO}$ emissions, this type of models may be too limited, then non-parametric response surfaces may be chosen as RBF or kriging (Seabrook et al. 2007). We will not detail this step of the calibration process in this paper, the reader may find more details on this topic in Castagné et al. (2008).

(v) optimizing the engine control parameters to meet the allocations. The problem may be formulated as a classical mathematical problem of optimization under constraints or as a multi-objective optimization (searching for compromises between antagonist objectives) as it is detailed in the following section. For this classical approach, the optimization is performed one OP after the other, considering the allocations of each OP as constraints. We refer in the following to this optimization problem as the OP optimization problem.

When the optimal settings are found, the last step consists in integrating them in the reference engine maps (if available) or building maps from these settings on the whole engine operating domain (see Fig. 7 for examples of engine maps). For the drivability of the target vehicle and because sharp evolution of air loop parameters are not easily feasible during transient, it is necessary to provide smooth engine maps. Thus, the settings are often moved away from their optimal values in order to build 
smooth engine maps, especially for air loop parameters. This smoothing process can be performed with respect to local constraints (such as maximum gradients), as well as to keep some predefined shapes. The difficulty of this step is thus to remain as close as possible to the local optima while preserving a smooth shape of the map, in order to keep all the benefits of the optimization work and satisfy the targets. This delicate step is usually time-consuming and is likely to deteriorate the work performed during the optimization phase by providing sub-optimal engine tunings. This is the motivation for our proposal of an integrated method of map optimization described in Sect. 4.

\subsection{The OP optimization problem formulation}

The OP optimization problem consists in minimizing some engine responses under constraints on other engine responses whereas the engine control parameters are kept inside the domain usually modeled by linear constraints coupling the parameters (see Fig. 2). It leads to the multi-objective optimization problem

$$
\min _{p \in \mathbb{R}^{N}}\left\{f_{1}(p), f_{2}(p), \ldots, f_{n}(p)\right\}
$$

subject to

$$
\begin{aligned}
\ell \leq A p & \leq u, \\
f_{j}(p) & \leq s_{j}, \quad j \in[1, d],
\end{aligned}
$$

and $d \leq n$. A classical example in turbo-diesel engine is the two-objective problem in which one attempts to minimize the particulate emissions and the $\mathrm{NO}_{\mathrm{x}}$ emissions under constraints on fuel consumption ( $\mathrm{CO}_{2}$ emissions), on $\mathrm{CO}$ and $\mathrm{HC}$ emissions and engine noise level.

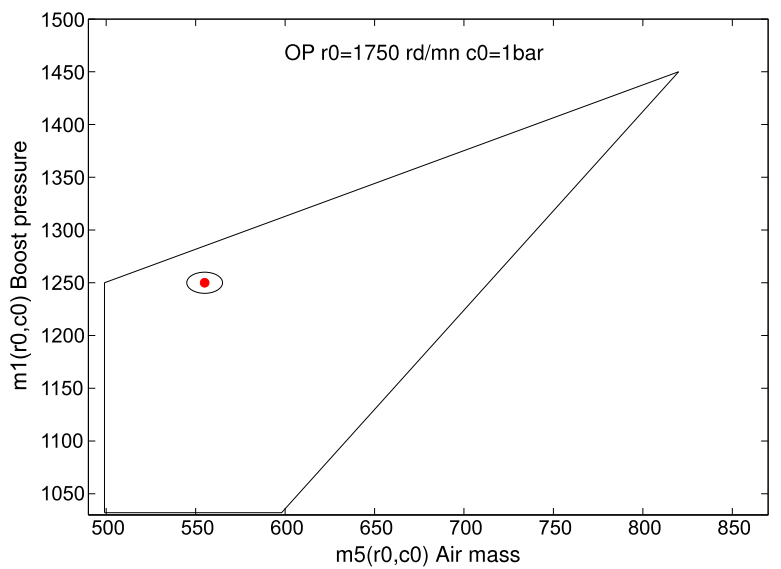

Fig. 2 Projection of the domain of parameter variations in the (boost pressure, air mass) plane. The limits of the domain are generally modeled by linear constraints on the engine control parameters. The ellipse indicates the parameter dispersions (here projected on the 2D space) modeled by a multidimensional Gaussian probability law. The ellipsoïd should remain in the engine physical domain in order to avoid that any control parameter takes some value outside this domain after the implementation in the control unit 
Traditionally, the multi-objective problem (1) is replaced by a constrained optimization problem where only one of the objectives is actually minimized, the other being constrained to remain below the prescribed thresholds. In other words, if the top priority is given to the $i_{0}$-th objective function, then what we perform is

$$
\min _{p \in \mathbb{R}^{N}} f_{i_{0}}(p)
$$

subject to

$$
\begin{aligned}
\ell \leq A p & \leq u, \\
f_{j}(p) & \leq s_{j}, \quad j \in[1, d] .
\end{aligned}
$$

This constrained single-objective problem (2) is then solved by a classical SQP method run several times with different initial points to search for the global optimum.

\section{Solving the OP multi-objective optimization problem}

In this section, we present a method to solve the constrained multi-objective problem (1). It is based on the algorithm proposed by Igel et al. (2007), namely, the MultiObjective Covariance Matrix Adaptation Evolution Strategy method (MO-CMA-ES). The MO-CMA-ES method belongs to the class of evolution strategy methods which allow to solve global optimization problems. These methods are popular for their ability to find a global minimum but their slow convergence rate is often criticized. In Hansen and Ostermeier (1996) was proposed the CMA-ES method for optimization of real-space functions, based on an original mutation operator that increases the convergence rate thanks to the adaptation of the mutation distribution at each generation.

\subsection{CMA-ES method for single-objective optimization}

In the CMA-ES evolutionary algorithm, a new population $x^{(g+1)}$ is created from the current population $x^{(g)}$ by generating realizations of a Gaussian probability law

$$
x_{k}^{(g+1)} \sim \mathcal{N}\left(e^{(g)},\left[\sigma^{(g)}\right]^{2} C^{(g)}\right) .
$$

Each subscript $k \in[1, K]$ corresponds to an individual of the population. In the distribution law $\mathcal{N}$, the expected value $e^{(g)}$ is chosen to be

$$
e^{(g)}=\sum_{k=1}^{\mu} w_{k} x_{k: K}^{(g)}
$$

where the notation $x_{k: K}^{(g)}$ stands for the $k$-th best individual of the current population $x^{(g)}$ with respect to the function to be minimized, and

$$
\sum_{k=1}^{\mu} w_{k}=1 \quad \text { and } \quad 0 \leq w_{\mu} \leq w_{\mu-1} \leq \cdots \leq w_{1} \leq 1 .
$$


In doing so, we ensure that the next population is directed towards the best points.

An ideal choice for the covariance matrix would be the inverse of the Hessian matrix of the objective function. The idea proposed by Hansen is then to approximate this matrix as for a quasi-Newton method but without any computation of the derivatives. The update formula for the covariance matrix is then

$$
C^{(g+1)}=\left(1-c_{\operatorname{cov}}\right) C^{(g)}+c_{\operatorname{cov}} \sum_{k=1}^{\mu} w_{k}\left(\frac{x_{k: K}^{(g+1)}-e^{(g)}}{\sigma^{(g)}}\right)\left(\frac{x_{k: K}^{(g+1)}-e^{(g)}}{\sigma^{(g)}}\right)^{T},
$$

which amounts to adding to the current covariance matrix a $\mu$-rank term from the best $\mu$ individuals of the new population. An alternative update formula is proposed by Hansen, which takes into account not only the last iteration as in (5) but also all the previous iterations from the beginning.

In a similar fashion, an update formula of the standard deviation is built: the main idea is here to avoid that two consecutive iterations lead to a displacement of the barycenter $e^{(g)}$ of the population in the same direction, replacing these two iterations by one with a larger standard deviation would have been a better choice. Likewise, a small standard deviation is preferred to a large one which leads to two iterations with opposite displacements of the barycenter of the population. The reader is referred to Hansen and Ostermeier (1996) for more details. Figure 3 illustrates on a 2D single-objective optimization example the adaptation of the covariance matrix and of the standard deviation leading to an efficient evolution of the population toward the global minimum.

\subsection{MO-CMA-ES method for multi-objective optimization}

Adapting the CMA-ES method to multi-objective optimization requires the definition of new criteria to define what is a good and a bad individual in the selection step of the evolutionary algorithm (see Igel et al. 2007). Two criteria are implemented in MO-CMA-ES:

1. The first criterion is the Pareto dominance. Let us recall this notion.

Definition 1 An individual $x$ is said to dominate another individual $x^{\prime}$, which is symbolized by $x \prec x^{\prime}$, if and only if

$$
\begin{array}{ll}
\text { - } \forall i \in\{1, \ldots, n\}, & f_{i}(x) \leq f_{i}\left(x^{\prime}\right) ; \\
\text { - } \exists i \in\{1, \ldots, n\}, & f_{i}(x)<f_{i}\left(x^{\prime}\right) .
\end{array}
$$

Definition 2 The non-dominated subset ndom $(X)$ of a given set $X$ is defined as

$$
\operatorname{ndom}(X)=\left\{x \in X \mid \nexists x^{\prime} \in X, x^{\prime} \prec x\right\} .
$$

The elements of this subset are said to be Pareto-optimal points.

Additionally, the Pareto front is composed of the values taken by the objectives for all the Pareto optimal solutions. Examples of Pareto fronts are given in Fig. 6. 

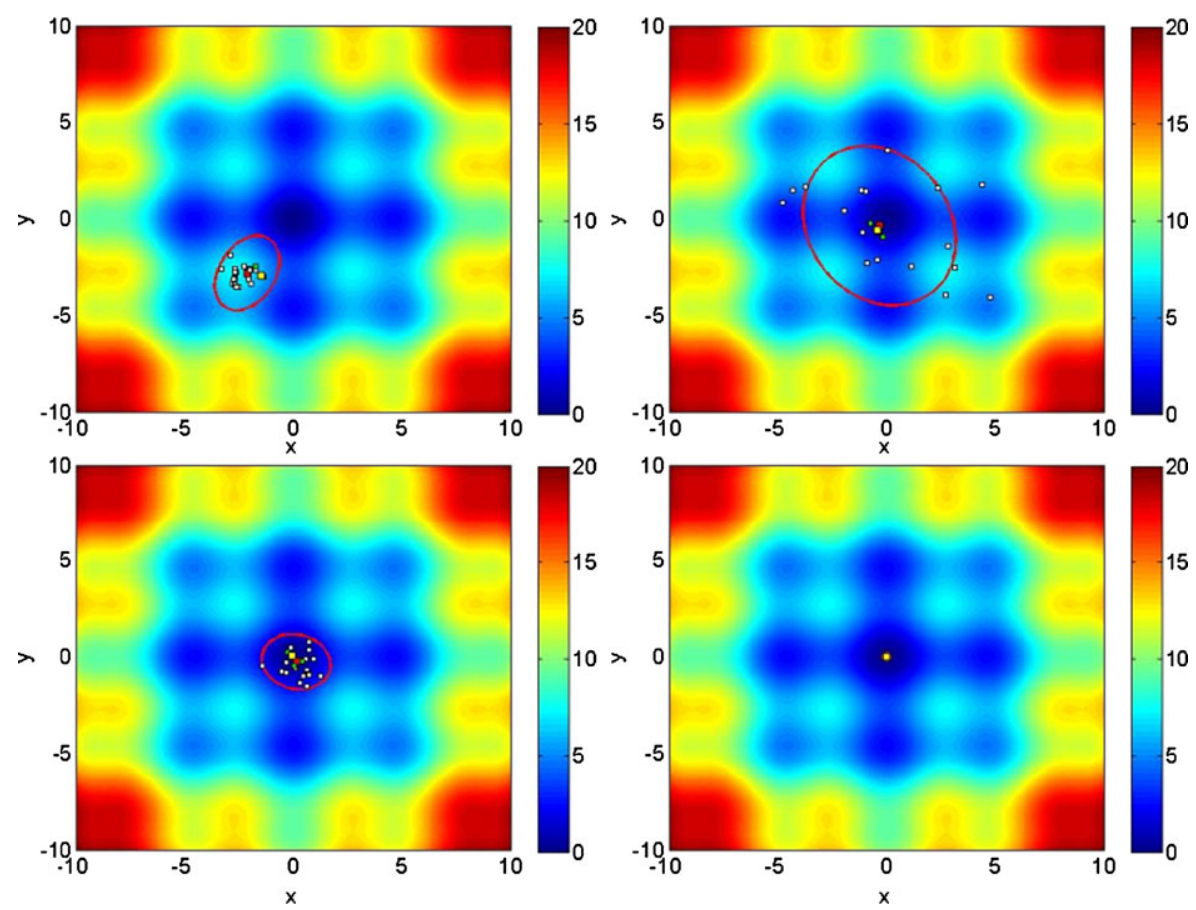

Fig. 3 An application of CMA-ES method on the analytical function $f(x, y)=\frac{x^{2}+y^{2}}{10}-\frac{1}{2}\left(\cos \left(\frac{2 \pi}{5} x\right)+\right.$ $\left.\cos \left(\frac{2 \pi}{5} y\right)\right)+3$. From top left to bottom right, the population at iterations 1, 5, 8 and 20 are represented. The ellipse represents the $95 \%$-isovalue of the Gaussian probability density of the mutation operator with the adaptive covariance matrix

The individuals of a given population $X$ are sorted by their non-dominance level. The notion of level is defined by induction as follows. First, we consider that the elements of $\operatorname{ndom}(X)$ have a non-dominance level $L=1$. Then, we introduce the points of level $L=2$ as the non-dominated points of the population $X$ without the non-dominated solutions. For $L \geq 1$, we thus obtain the recursive definition

$$
\begin{aligned}
\operatorname{dom}_{0}(X) & =X ; \\
\operatorname{ndom}_{L}(X) & =\operatorname{ndom}\left(\operatorname{dom}_{L-1}(X)\right) ; \\
\operatorname{dom}_{L}(X) & =\operatorname{dom}_{L-1}(X) \backslash \operatorname{ndom}_{L}(X) .
\end{aligned}
$$

2. The second criterion is a diversity criterion of the solutions to obtain an uniform repartition of the solutions along the Pareto front. A measure of the dominance hyper-volume at a non-dominated solution is defined by considering the surface of the rectangles defined in Fig. 4 (one rectangle for each non-dominated point).

Combining these two criteria allows to be sorted the individuals of the current population with respect to the multiple objectives and then to apply the CMA-ES update formula of the covariance matrix adaptation for the mutation. For more details on the MO-CMA-ES method, the reader is referred to Igel et al. (2007). 
Fig. 4 Contributing dominance hyper-volumes for the evaluation of the diversity criterion

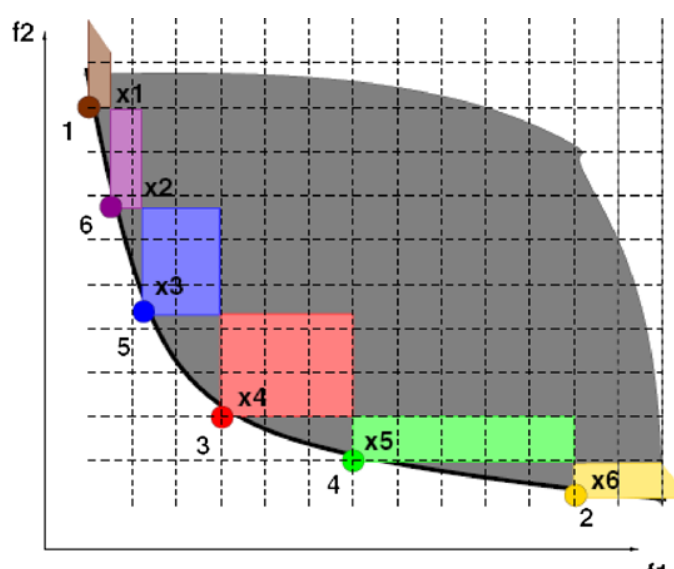

f1

\subsection{Application of MO-CMA-ES method to a real case of engine calibration}

In this section, we present some results obtained from a real dataset associated with a turbo-diesel engine. We consider the optimization problem (1) at a given engine operating point ( $1750 \mathrm{rd} / \mathrm{mn}-1 \mathrm{bar}$ ). Models of the engine responses have been built from experimental data, the tests being designed thanks to a classical D-optimal criterion: the models are cubic polynomials. Figure 5 illustrates the quality of the models at some validation points chosen randomly in the admissible domain (those points were not used for model building). An average value of the measurement error has been estimated from some repeated measurements. The model of $\mathrm{NO}_{\mathrm{x}}$ emissions matches very well the data within the error bars whereas the model of particulate emissions is less accurate: this is due to large error measurements for the engine response and a bad approximation of the measurement error when considering this error to be constant. Nevertheless, engine experts have validated this model considering that the trends were well modeled.

The optimization is performed on 6 engine control parameters: the main injection timing, the pilot fuel injection quantity, the pilot injection timing, the fuel injection pressure, the mass air flow and the boost pressure.

The optimization objectives are the particulate emissions and the $\mathrm{NO}_{\mathrm{x}}$ emissions, $\mathrm{CO}_{2}, \mathrm{HC}$ and $\mathrm{CO}$ emissions and the engine noise being constrained to remain smaller than a given threshold. Linear constraints defining physical limits of the domain of variations of the parameters are introduced. These linear constraints may take into account the parameter dispersions, these new constraints are denoted by robustness constraints hereafter.

Thus, the multi-objective optimization algorithm has been modified to deal also with linear and nonlinear inequality constraints: they have been introduced via a $l_{1}$ penalty term added to the objectives (if the $j$-th constraint is not satisfied, the misfit with the bound is added to the objective functions, the penalty weight varying during the iterations).

The results are presented in Fig. 6: two optimizations are compared, one without taking into account the parameter dispersions and one with the robustness constraint 


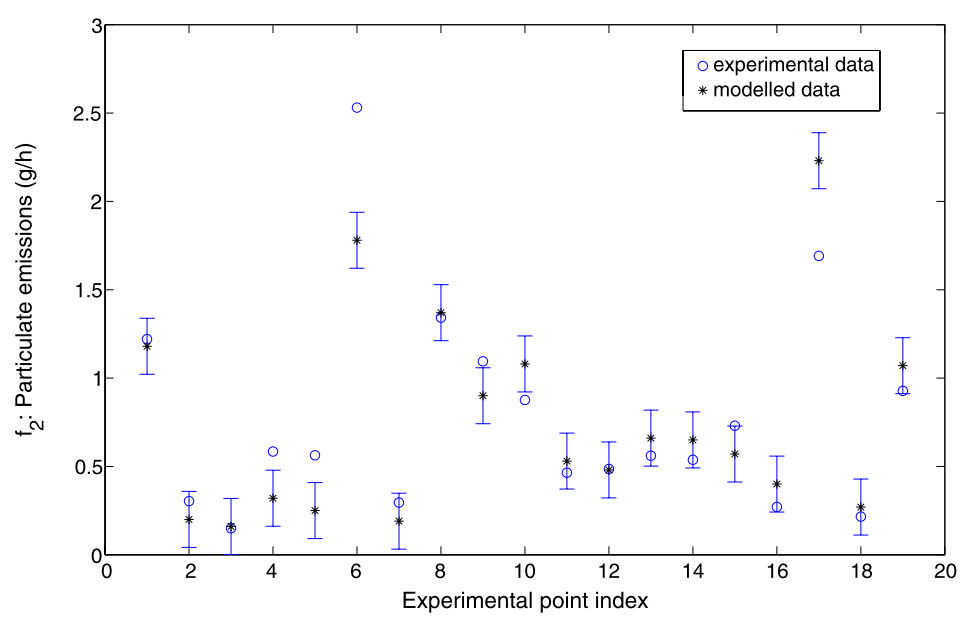

(a) Particulate emissions

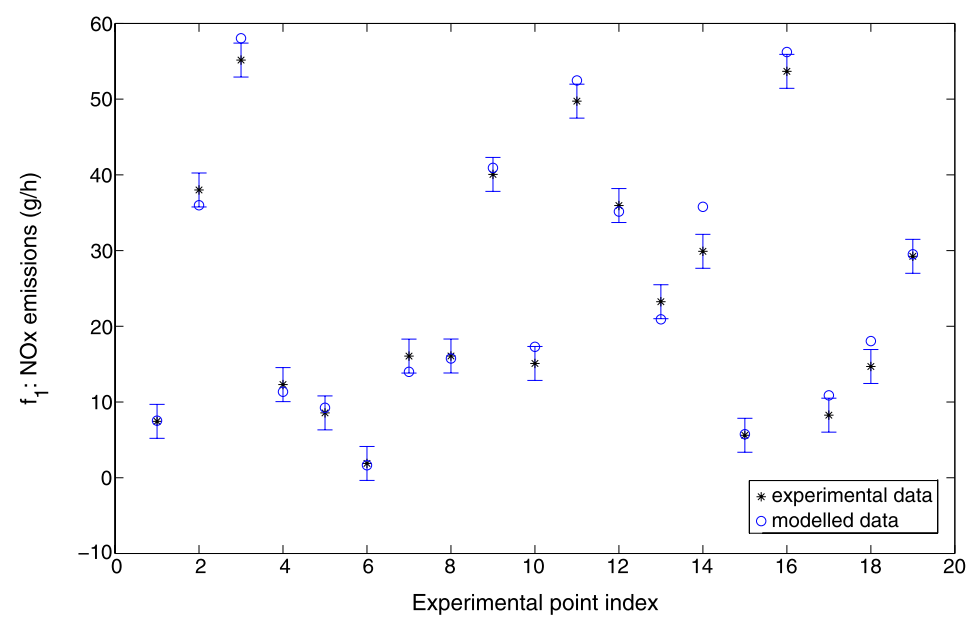

(b) $\mathrm{NO}_{\mathrm{x}}$ emissions

Fig. 5 Comparison of experimental data and model predictions for given parameter sets (those points were not used for model building) at $\mathrm{OP} 1750 \mathrm{rd} / \mathrm{mn}-1$ bar for $\mathrm{NO}_{\mathrm{x}}$ (a) and particulate emissions (b) in $\mathrm{g} / \mathrm{h}$. Error bars correspond to measurement error estimates from some repeated experiments

which consists in taking into account the possible dispersion of the engine control parameters when applied on the vehicle. This dispersion is modeled by an uncorrelated Gaussian probability law, thus by one standard deviation by parameter. As shown in Fig. 2, a vicinity of the engine setting is defined and this vicinity should remain inside the physical limits. The impact of this constraint is noticeable in Fig. 6 by an important shift of the Pareto front and a modification of its shape. The points indicated in pink are associated with successive runs of a local single-objective optimization with different initial points and with a weighted sum of the two objectives (different weight values have been tested). We notice that these optimizations lead to 


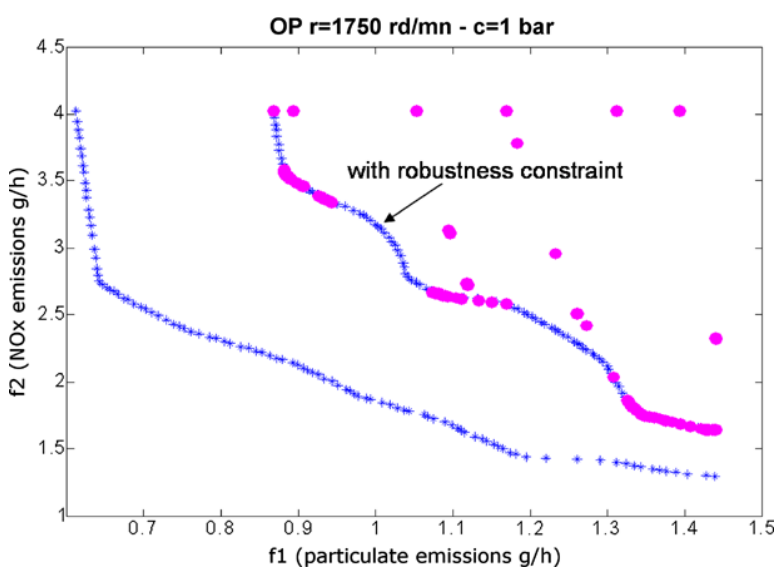

Fig. 6 Pareto fronts (crosses) of the bi-objective optimization of particulate emissions and $\mathrm{NO}_{\mathrm{x}}$ emissions (in $\mathrm{g} / \mathrm{h}$ ) obtained with the MO-CMA-ES method for the OP $1750 \mathrm{rd} / \mathrm{mn}-1 \mathrm{bar}$. The front at the right side of the figure is associated with an optimization taking into account the parameter dispersion (robustness constraints that ensure the effective engine control parameter values to remain inside the engine physical domain). The other front results from an optimization without these constraints. The dots indicate solutions obtained by single-objective optimizations (weighted sum of the two objectives) associated with different initial points and different penalty weights

points not located on the Pareto front: these points are some local minima. Moreover, as expected, the points obtained on the Pareto front are located on the convex parts only.

The MO-CMA-ES method has performed on this example 3 times more evaluations of the objective functions than the local optimization with different initial points and different weights. Even if this performance is already very encouraging, some work on the stopping criteria may reduce the number of evaluations of MO-CMAES.

Finding a $\mathrm{NO}_{\mathrm{x}} /$ particulate emissions compromise is a classical calibration problem for diesel engine. Engine experts were thus very interested in the different compromises between the two objectives that have been obtained by the multi-objective optimization method. We clearly observe 3 interesting valleys on the Pareto front with a significant decrease of $\mathrm{NO}_{\mathrm{x}}$ emissions for a small increase of particulate emissions. This method allows then a finer choice of the calibration control parameter set than a classical method based on a mono-objective formulation.

\section{Solving the map optimization problem}

In the classical calibration process described in Sect. 2, the engine map building in the smoothing step is delicate and time-consuming. In this section, we propose an alternative method that consists in directly optimizing the cumulated engine responses over the cycle via deformations of the engine maps themselves instead of optimizing individually the selected OP and building afterward the engine maps by the smooth- 
ing step. The map optimization problem is formulated as

$$
\min _{m: \mathbb{R}^{2} \rightarrow \mathbb{R}^{M}}\left\{\int_{0}^{T} F_{i}(r(t), c(t), m(r(t), c(t))) d t\right\}_{1 \leq i \leq n}
$$

subject to

$$
\begin{aligned}
\ell(r, c) \leq A m(r, c) \leq u(r, c), \quad(r, c) \in \mathbb{R}^{2}, & \\
\int_{0}^{T} F_{j}(r(t), c(t), m(r(t), c(t))) d t & \leq S_{j}, \quad j \in[1, d] .
\end{aligned}
$$

Here,

- $t \in \mathbb{R} \mapsto(r(t), c(t)) \in \mathbb{R}^{2}$ denote the (known) trajectory of the cycle within the engine speed-load domain;

- $(r, c) \in \mathbb{R}^{2} \mapsto m(r, c) \in \mathbb{R}^{M}$ are the $2 \mathrm{D}$ engine maps; the components of $m$ are called engine control parameters;

- $F_{i}$ is the model of the engine response $i$, as a function of the engine control parameters but also of the speed and load.

The objectives to be minimized (or constrained) are the engine responses cumulated on the considered driving cycle: the cumulated responses are here (see (10)) the weighted sums of the local models defined at chosen representative OP but if global models of the engine responses are available (models including engine speed and load dependency), a finer optimization may be performed with a fine sample of the integrals in the original optimization problem (9). Additional smoothing constraints such as global smoothing constraints (to preserve the regularity of the original maps) or more local constraints (for example limits on the gradients of the maps) can also be introduced.

Using a quadrature rule involving the OPs $\left(r_{q}, c_{q}\right), q \in\left[1, N_{\mathrm{OP}}\right]$, to approximate the continuous integral (9a), we end up with the discrete version

$$
\min _{m: \mathbb{R}^{2} \rightarrow \mathbb{R}^{M}}\left\{\sum_{q=1}^{N_{\mathrm{OP}}} \xi_{q} F_{i}^{(q)}\left(m\left(r_{q}, c_{q}\right)\right)\right\}_{1 \leq i \leq n}
$$

subject to

$$
\begin{aligned}
& \ell\left(r_{q}, c_{q}\right) \leq A m\left(r_{q}, c_{q}\right) \leq u\left(r_{q}, c_{q}\right), \quad q \in\left[1, N_{\mathrm{OP}}\right], \\
& \sum_{q=1}^{N_{\mathrm{OP}}} \xi_{q} F_{j}^{(q)}\left(m\left(r_{q}, c_{q}\right)\right) \leq S_{j}, \quad j \in[1, d] .
\end{aligned}
$$

\subsection{Modeling the engine maps}

In the formulations (9) and (10), minimization has to be carried out with respect to a function $m(.,):. \mathbb{R}^{2} \rightarrow \mathbb{R}^{M}$, which is a continuous object. In order to fully discretize 
$\mathrm{m} 2$ : Main injection timing
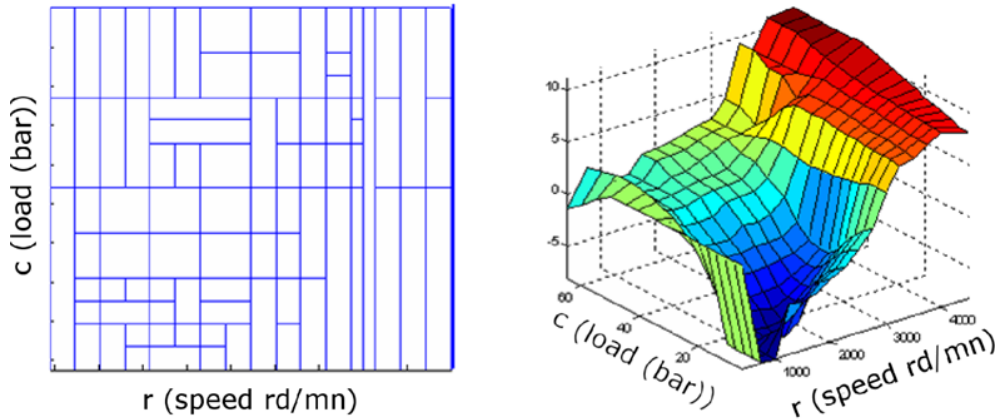

$\mathrm{m} 1$ : Boost pressure
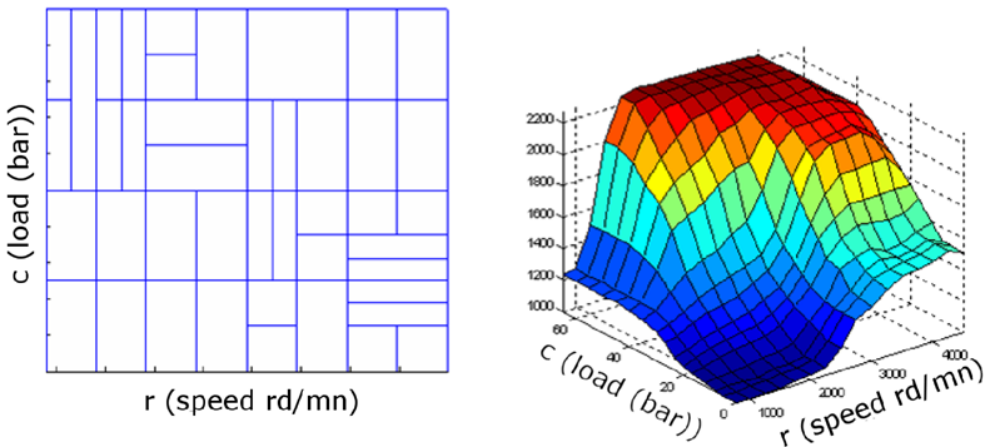

Fig. 7 Examples of LoLiMoT parameterization of engine map. Left: patching of the domain. A local linear model is defined on each patch. Right: the resulting surfaces modeled by LoLiMoT for two different engine control parameters from given discrete reference maps

the problem, we have to consider a parameterization of the engine maps that must be flexible enough to model the very different shapes of engine map surfaces (Fig. 7) and that should not require too many parameters to limit the number of unknowns in the optimization process. LoLiMoT models (Nelles 2001; Hafner and Isermann 2001) seem to be a good compromise between flexibility, accuracy and complexity: some very simple local models (linear or bilinear) are combined by a weighted sum

$$
m(r, c)=\sum_{\lambda=1}^{\Lambda} \widehat{m}_{\lambda}(r, c) \Phi_{\lambda}(r, c),
$$

with

$$
\widehat{m}_{\lambda}(r, c)=\theta_{0, \lambda}+\theta_{r, \lambda} r+\theta_{c, \lambda} c .
$$

The weights $\Phi_{\lambda}(r, c)$ are normalized Gaussian functions defined as

$$
\Phi_{\lambda}(r, c)=\frac{\mathfrak{N}_{\lambda}(r, c)}{\sum_{\kappa=1}^{\Lambda} \mathfrak{N}_{\kappa}(r, c)},
$$


with

$$
\mathfrak{N}_{\lambda}(r, c)=\exp \left\{-\frac{1}{2 \alpha}\left[\left(\frac{r-r_{\lambda}^{0}}{\sigma_{\lambda}^{r}}\right)^{2}+\left(\frac{c-c_{\lambda}^{0}}{\sigma_{\lambda}^{c}}\right)^{2}\right]\right\} .
$$

Their purpose is to control the degree of smoothness of the global surface. $\alpha$ is a global smoothing parameter. As far as the coefficients $\theta_{0, \lambda}, \theta_{r, \lambda}$ and $\theta_{c, \lambda}$ are concerned, each of them is a vector of size $M$. Therefore, the continuous function $m(.,$. has been replaced by a vector

$$
p=\left\{\theta_{0, \lambda}, \theta_{r, \lambda}, \theta_{c, \lambda}\right\}_{1 \leq \lambda \leq \Lambda}
$$

whose size is $N=3 M \Lambda$. Substituting (11) into (10) brings us back to problem (1).

The advantage of the LoLiMoT representation (11) lies in its ability to adaptively refine the surface: the patching associated with the definition domains of the local models may be refined during the optimization process. A finer patching allows a finer optimization (the number of degrees of freedom being increased) but may lead to a cumbersome optimization of a large number of parameters. The parameterization, namely the patch definition for LoLiMoT description of the maps should reflect the degree of smoothness the user expects for the maps: for some parameters like boost pressure, the map should remain smooth, for others like main injection timing, the smoothing constraint is not as strong. An example of the LoLiMoT parameterization in the context of engine tunings is displayed in Fig. 7.

\subsection{Application of map optimization on a real case}

The map optimization has been applied on the turbo-diesel engine application described in Sect. 3.3. The optimization problem is here defined as a constrained singleobjective optimization problem on the whole driving cycle whereas a two-objective formulation was used for the local optimization problem at a fixed OP. The cumulated particulate emissions on the cycle are minimized and the cumulated $\mathrm{CO}_{2}, \mathrm{CO}, \mathrm{HC}$ and $\mathrm{NO}_{\mathrm{x}}$ emissions are constrained to remain under thresholds (given in Table 1). The unknowns of the optimization problem are the 378 LoLiMoT parameters describing the 6 initial engine maps. Linear constraints are added to take into account the limits of the physical domain for each point of the engine maps. The obtained results are listed in Table 1 and the optimized engine maps are displayed in Fig. 8. These engine maps have been run at the test bench and particulate emissions have been measured, those measures are displayed in Fig. 9.

Table 1 Results of map optimization on a diesel engine application

\begin{tabular}{lcccc}
\hline & Initial maps & Optimized maps & Upper bounds & Error bar \\
\hline Particulate $(\mathrm{g} / \mathrm{km})$ & 0.128 & 0.090 & - & 0.003 \\
$\mathrm{NO}_{\mathrm{x}}(\mathrm{g} / \mathrm{km})$ & 0.219 & 0.240 & 0.241 & 0.025 \\
$\mathrm{HC}(\mathrm{g} / \mathrm{km})$ & 0.137 & 0.150 & 0.150 & 0.014 \\
$\mathrm{CO}(\mathrm{g} / \mathrm{km})$ & 0.815 & 0.847 & 0.896 & 0.039 \\
$\mathrm{CO}_{2}(\mathrm{~g} / \mathrm{km})$ & 168.89 & 164.40 & 168.89 & 1.055 \\
\hline
\end{tabular}




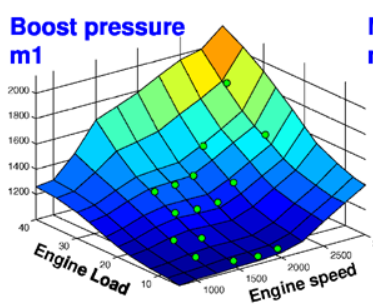

Pilot injection timing
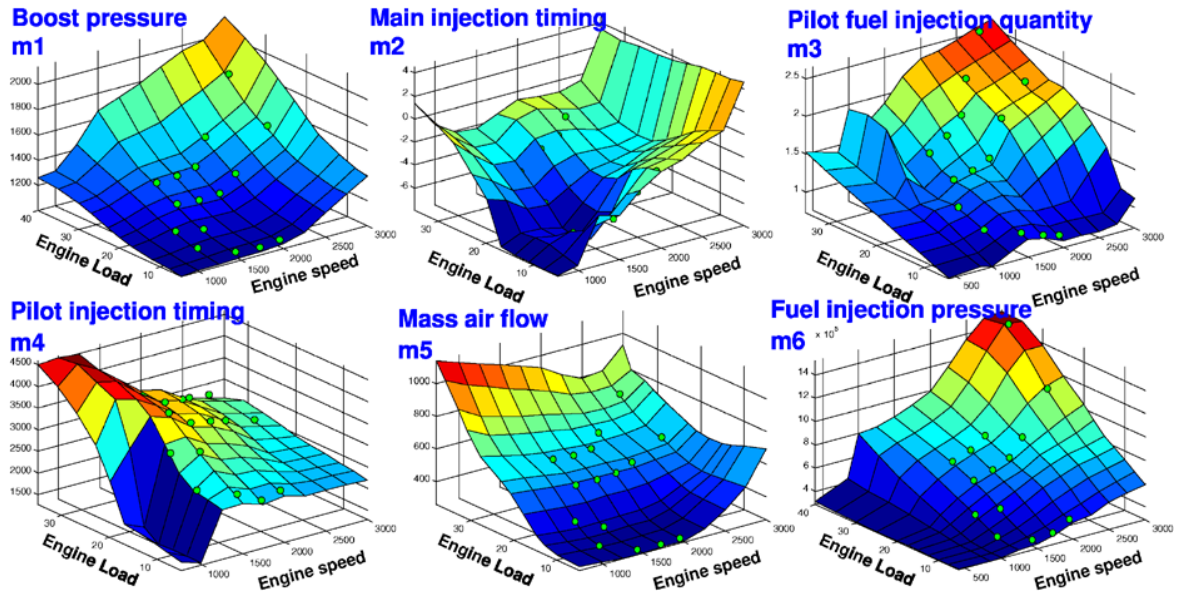

Fig. 8 Optimal engine maps obtained by the map optimization on the NEDC driving cycle. 378 LoLiMoT parameters are necessary to model the 6 maps with the expected regularity. The green points indicate the engine operating points for which models of engine responses are available

Fig. 9 Comparison of measures of particulate emissions at the test bench for the initial engine maps (top) and the optimal ones (bottom) obtained by the proposed map optimization method

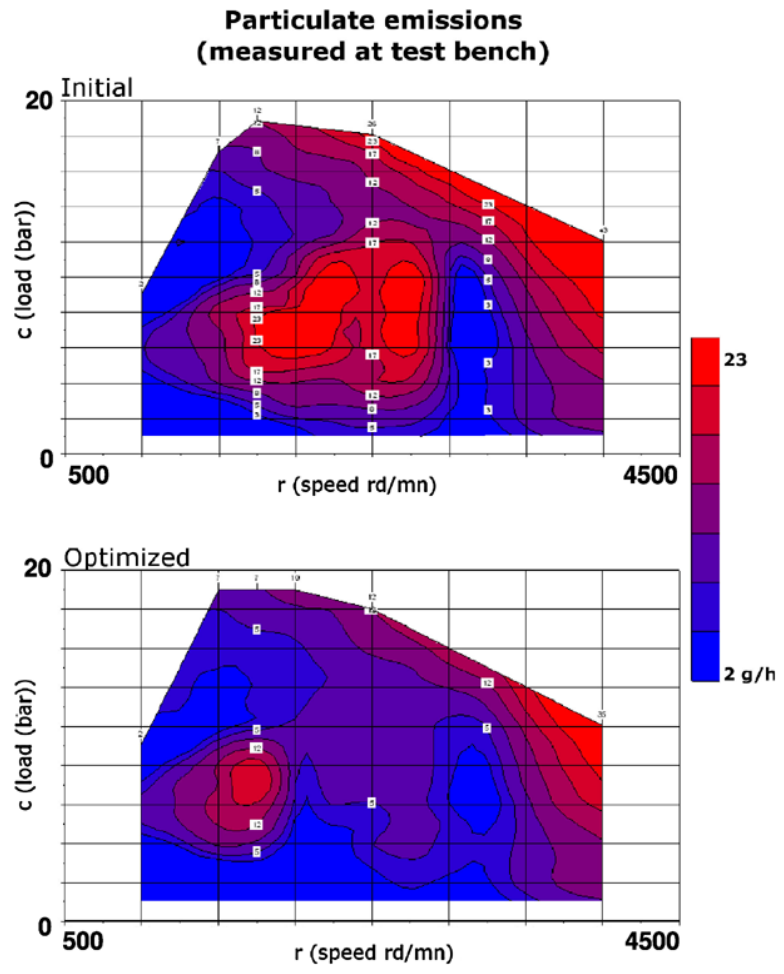

\section{(measured at test bench)}

Table 1 shows that the objectives of optimization are reached: the particulate emissions are minimized whereas all the other cumulated engine responses remain under the constraint thresholds. Moreover the chosen map parametrization allows us 
to match the smoothness constraints, which is crucial for the feasibility of the engine tunings. The comparison of the particulate emissions obtained at test bench with the initial maps and the optimized on Fig. 9 illustrates the obtained gain: in the area covered by the optimized driving cycle (under $3000 \mathrm{rd} / \mathrm{mn}$ and under $10 \mathrm{bar}$ ), the emissions are obviously minimized. In the other parts of the operating domain, the differences are the result of the smoothing constraints applied on the maps introduced in the optimization formulation by means of second derivatives minimization. In contrast to successive local OP optimizations, the global optimization offers the flexibility to increase slightly the particulate emissions locally if it is necessary to match the smoothing constraints. It is the trend we observe on Fig. 9 in the area located around $1750 \mathrm{rd} / \mathrm{mn}-7$ bar.

\section{Conclusions}

Throughout this contribution, we have demonstrated the relevance of the two approaches proposed for engine calibration. By extending the standard local singleobjective formulation in two directions, namely, the local multi-objective formulation and the global single-objective formulation, we seek to provide engine experts with a more powerful tool that enables them to achieve better results.

The technical challenges we had to face were solved by existing dedicated algorithms. For the local multi-objective approach, the constrained multi-objective optimization was successfully carried out by the MO-CMA-ES method, which we slightly modified to handle inequality constraints. The performance of this method on the considered real case application is very encouraging, in the sense that the Pareto front obtained gives worthy information on the possible compromises between antagonist engine responses, such as particulate emissions and $\mathrm{NO}_{\mathrm{x}}$ emissions of a diesel engine. As far as the global single-objective approach is concerned, the fact that it rests upon a cycle optimization of the engine maps instead of a pointwise optimization allows us to avoid the cumbersome step of map smoothing in classical engine calibration process. The numerical results testify to a notable gain in efficiency thanks to intrinsic smoothing constraints introduced via the LoLiMoT parameterization.

An upcoming study for the map optimization will be to apply a multi-objective approach as for the OP optimization. Here, the difficulty lies in the large size of the parameter space (500 parameters to describe all the engine maps). Another improvement in this approach will be the use of global models of engine responses (defined on the whole engine operating domain instead of the local models at a limited number of OP): this optimization is expected to yield finer results.

Acknowledgements The authors are grateful to thank Yohan Bentolila, Michel Castagné, Fabien Chaudoye, Roland Dauphin, Adrien Hallé and Frédéric Nicolas for introducing us to the engine calibration problem and for supplying us with the real dataset used in this study. We also wish to thank the two anonymous referees for their constructive comments, which helped us to improve this paper. 


\section{References}

Buzy A, Cotte P, Visconti J (2005) Online adaptive DOEs and global modelling for diesel engines and DPF calibration. In: IAV Berlin conference: design of experiments in engine development. Expert Verlag, Ehningen, pp 133-146

Castagné M, Bentolila Y, Chaudoye F, Hallé A, Nicolas F, Sinoquet D (2008) Comparison of engine calibration methods based on design of experiments. Oil Gas Sci Technol, Rev l'IFP, 63(4):563-582

Das I, Dennis JE (1998) Normal-boundary intersection: a new method for generating the Pareto surface in nonlinear multicriteria optimization problems. SIAM J Optim 8(3):631-657

Hafner M, Isermann R (2001) The use of stationary and dynamic emission models for an improved engine performance in legal test cycles. In: International workshop on modeling, emissions and control in automotive engines, Salerno, Italy

Hansen N, Ostermeier A (1996) Adapting arbitrary normal mutation distributions in evolution strategies: the covariance matrix adaptation. In: Proceedings of the 1996 IEEE international conference on evolutionary computation, pp 312-317

Igel C, Hansen N, Roth S (2007) Covariance matrix adaptation for multi-objective optimization. Evol Comput 15(1):1-28

Leyffer S (2009) A complementarity constraint formulation of convex multiobjective optimization problems. INFORMS J Comput 21(2):257-267

Nelles O (2001) Nonlinear system identification: from classical approaches to neural networks and fuzzy models. Springer, Berlin

Neßler A, Haukap C, Roepke K (2006) Global evaluation of the drivability of calibrated diesel engine maps. In: Proceedings of the 2006 IEEE conference on computer aided control systems design, $\mathrm{Mu}-$ nich, Germany, October 4-6, pp 2683-2688

Röpke K, Gaitzsch R, Haukap C, Knaak M, Knobel C, Neßler A, Schaum A, Schoop U, Tahl S (2005) DoE-design of experiments, methoden und anwendungen in der Motorenentwicklung. Verlag Moderne Industrie, Augsburg

Sampson D, Sheridan L (2007) Recent developments in techniques for model-based calibration. In: Röpke K (ed) Design of experiments (DoE) in engine development III, Berlin. Expert Verlag, Ehningen, pp 45-55

Schloßer A, Linssen R, Bozelie P (2007) Model-based calibration of SI-engines map optimization. In: Röpke K (ed) Design of experiments (DoE) in engine development III, Berlin. Expert Verlag, Ehningen, pp 145-164

Schmied A (2003) A global constrained optimization algorithm for engine calibration. In: Bliek C et al (eds) Selected papers of the first international workshop on global optimization and constraint satisfaction, COCOS 2002. Lecture notes in computer science, vol 2861. Springer, Berlin, pp 111122

Seabrook J, Cornwell R, Mortimer P, Edwards S (2007) A calibration methodology to ensure low variation in emissions between engines in production. In: IAV/Haus der Technik 4th conference on DOE in engine development, Berlin. Expert Verlag, Ehningen, pp 34-44 